Barcelona, Spain. The cause of the chorea was streptococcal infection in 2, post-head injury (1), and unknown in 2. Therapy was continued for 3 to 36 months; it was discontinued in 1 because of an allergic cutaneous rash. (Roig $M$ et al. Carbamazepine: An alternative drug for the treatment of nonhereditary chorea. Pediatrics Sept 1988; 12 (pt 2): 492-495).

COMMENT. Prednisone has also been advocated in the treatment of Sydenham's chorea. Kelts and Harrison, reporting at the recent 17 th annual meeting Child Neurology Society in Halifax, found predisone beneficial in 9 cases; an initial average dose of $1.8 \mathrm{mg} / \mathrm{kg} /$ day begun within 2 weeks of onset of chorea was tapered over 2 to 6 months.

Despite the self-limiting nature of the involuntary movements, they are frequently incapacitating and warrant treatment. If low to moderate doses of phenobarbital are ineffective, a trial of carbamazepine appears to be worthwhile, and predisone in resistant cases. Paradoxically, choreoathetosis or dystonia may occur as a side-effect of carbamazepine treatment in epileptic patients.

\title{
CEREBRAL TRAUMA
}

\section{SEVERE HEAD INJURIES}

The clinical predictors of severe head trauma in 55 children, 1 to 15 years of age, were compared with CT scan findings at the Dept of Pediatrics, Medical College of Wisconsin and Children's Hospital of Wisconsin, Milwaukee, WI. Severity of head trauma was determined according to the presence or absence of clinical variables, including altered mental states, duration of loss of consciousness or $5 \mathrm{~min}$, vomiting, headache, focal neurologic deficit, seizure, and soft-tissue injury. Injury was considered severe if one or more of the following variables were present: altered mental status, increased intracranial pressure, and seizure or focal neurologic deficit. Thirty-seven ( 848 ) of 44 patients with severe head trauma had a positive CT scan. Six (138) with a Glasgow Coma Scale (GCS) score of 12 or greater had abnormal CT scans. All patients with mild or moderate head trauma had normal CT scans. Historical information and clinical examination were the most accurate predictors for abnormal CT scans regardless of GCS scores. (Hennes $\mathrm{H}$ et al. Clinical predictors of severe head trauma in children. AJDC Oct 1988; 142:1045-1047).

COMMENT. The GCS may have limited application in the evaluation of acute head trauma in the pediatric population. A classification based on clinical findings more accurately identifies the severity of head trauma and the need for CT scans. 
A further clinical objective evaluation of children with acute head injury includes tests of neuropsychological function. Investigators from the Dept Pediatrics, Univ of Maryland, Baltimore, report cognitive deficits immediately after closed head injury in adolescents that may interfere with school, home and peer activities ( Oct $1988 ; \underline{142}: 1048$ ).

\section{LEARNING AND BEHAVIOR}

\section{SUCROSE, MOTOR ACTIVITY, AND LEARNING}

The effects of sugar (sucrose) on the behavior of 30 preschool children ( 20 boys and 10 girls, mean age 5 years 4 mos) and 15 elementary school children ( 6 boys and 9 girls, mean age 7 yrs 2 mos) were investigated by psychologists from Colorado State University, Fort Collins, CO, and the Univ of Mississippi Med Cntr, Jackson, MS. Parents and teachers questioned before the study complained that the child was behaviorally sensitive to sugar in approx 508 of subjects. Two preschool children had been considered hyperactive by the school director. A basic breakfast included a $4 \mathrm{oz}$ orange flavored drink of high sucrose content (50g), low-sugar (6.25g) or aspartame (122 mg), randomly selected, 5 days on each, using a double-blind control design. The mean sucrose intakes for the high, low, and "control" aspartame conditions were 2.26, 0.28, and $0.00 \mathrm{~g} / \mathrm{kg}$, respectively, and the total carbohydrate contents of breakfast averaged $3.95,1.88$, and $1.54 \mathrm{~g} / \mathrm{kg}$, respectively.

On cognitive measures, girls made significantly more errors on a paired-associate learning task performed 20-30 min following a high-sugar content breakfast when compared to a low-sugar meal, whereas boys were unaffected. On global ratings, younger preschool children were affected differently than older children. On an Abbreviated Conners Teacher Rating Scale completed before lunch, both boys and girls were more active in behavior after the high sugar meal than that of low sugar content. Measures of behavior by observation for fidgetiness, change in activity, running, vocalization and aggressiveness and other cognitive measures involving matching and academic tasks failed to demonstrate changes after sugar ingestion. (Rosen LA et al. Effects of sugar (sucrose) on children's behavior. $\mathrm{J}$ Consulting Clin Psychol 1988; $56(4): 583-589$ ).

COMMENT. Evidently, the effects of sugar on children's behavior is not yet resolved. This study demonstrates significant adverse effects although the authors conclude that these are minimal in degree. Certain limitations of the study design are admitted: 1. The sugar challenge dose was the same for all subjects and younger and smaller children, affected differently, received larger amounts 